\title{
Automatic multiscale vascular image segmentation algorithm for coronary angiography
}

\author{
Adrián Carballal $^{\mathrm{a}, \mathrm{b}}$, Francisco J. Novoa ${ }^{\mathrm{a}}$, Carlos Fernández-Lozano ${ }^{\mathrm{a}, \mathrm{b}}$, \\ Marcos García-Guimaraes ${ }^{\mathrm{b}, \mathrm{c}}$, Guillermo Aldama-López ${ }^{\mathrm{b}, \mathrm{c}}$, Ramón \\ Calviño-Santos ${ }^{\mathrm{b}, \mathrm{c}}$, José Manuel Vázquez-Rodríguez ${ }^{\mathrm{b}, \mathrm{c}}$, Alejandro Pazos ${ }^{\mathrm{a}, \mathrm{b}}$ \\ ${ }^{a}$ Computer Science Department, Faculty of Computer Science, University of A Coruña, A Coruña \\ 15071, Spain \\ ${ }^{b}$ Instituto de Investigación Biomédica de A Coruña (INIBIC), Complexo Hospitalario \\ Universitario de A Coruña (CHUAC), A Coruña 15006, Spain \\ ${ }^{c}$ Department of Cardiology, Complexo Hospitalario Universitario de A Coruña (CHUAC), A \\ Coruña, Spain
}

\begin{abstract}
Cardiovascular diseases, particularly severe stenosis, are the main cause of death in the western world. The primary method of diagnosis, considered to be the standard in the detection and quantification of stenotic lesions, is a coronary angiography. This article proposes a new automatic multiscale segmentation algorithm for the study of coronary trees that offers results comparable to the best existing semi-automatic method. According to the state-of-the-art, a representative number of coronary angiography images that ensures the generalisation capacity of the algorithm has been used. All these images were selected by clinics from an Haemodynamics Unit. An exhaustive statistical analysis was performed in terms of sensitivity, specificity and Jaccard. Algorithm improvements imply that the clinician can perform tests on the patient and, bypassing the images through the system, can verify, in that moment, the intervention of existing differences in a coronary tree from a previous test, in such a way that it could change its clinical intra-intervention criteria.
\end{abstract}

\section{Keywords}

Multiscale segmentation; Coronary disease; Stenotic lesions; Angiographies segmentation 


\section{Introduction}

Cardiovascular diseases (CVD) are the number one cause of death globally. An estimated 17.9 million people died from CVDs in 2015, representing $31 \%$ of all global deaths. Of these deaths, an estimated 7.4 million were due to coronary heart disease and 6.7 million were due to stroke. Over three quarters of CVD deaths take place in lowand middle-income countries. Out of the 16 million deaths under the age of 70 due to non-communicable diseases, $82 \%$ are in low and middle income countries and $35 \%$ are caused by CVD [1].

The most used diagnostic technique at present, and that which is used for evident symptoms of cardiovascular problems, is an angiography obtained through catheterisation [2],[3]. This procedure is recommended in patients with a high probability of coronary heart disease. In these cases, cardiologists analyse the angiography images, establish a diagnosis for the disease and even anticipate its prognosis, depending on the severity and extent of the coronary disease.

Due to the importance of this type of test and its implications on public health, researchers such as O'Brian and Ezquerra [4] have been working on the automatic segmentation of the coronary tree since the mid 1990s. Since then, a large number of processing techniques have increased to efficiently segment the coronary tree, as can be verified in the comparative studies of Kirbas and Quek [5], published in 2004, and the studies of Novoa et al. [6], published in 2011. In this last study, it was concluded that the algorithm with the best results was that of Poon et al. [7]. However, this technique has the downside of a very long execution time, to the order of several minutes for an average sized image, and its semi-automatic nature requires human intervention. What is more, semi-automatic arterial segmentation has the issue of the existence of an interand intra-observer variable. Even though it is a value that can be minimised, as has been documented in several studies and publications, it is not possible to completely eliminate it [8]. Lastly, the efficiency of these semi-automatic algorithms is completely dependent on the experience of a clinical expert, and largely eliminates the generalisation capacity of this algorithm.

This article presents a new coronary tree segmentation technique that is completely automatic and efficient, in terms of execution time. Its results surpass those obtained by Poon et al. [7] in precision, a state of the art technique that was previously mentioned, and, according to several state of the art reviews, which currently offer the best results for this type of medical image.

\section{Background}

In the field of processing coronary angiographies, segmentation algorithms have to solve very complex problems, such as the noise that angiography capture devices register, the spontaneous movements of the patient and of his/her internal organs, bifurcations, different level blood-vessel crossings, stenotic lesions, etc. Due to all of this, it is very difficult to develop a technique that behaves appropriately in all of these situations.

Over the years, may studies have come into existence that present algorithms whose aim is to offer the user a reliable segmentation based on the data entry set within a reasonable period of time. However, many methods offer only one of these two characteristics. An example of this fact are Random Walk methods [9], which are one of the fastest, in general terms, of all the developments at present. However, they suffer from problems of false positives and are unable to completely ignore the noise of the 
input image. On the other hand, the active contour methods [10] are less sensitive to random noise, but the required execution time is much longer than that of the multiscale methods. Algorithms based on artificial neuron networks [11],[12] have experienced a significant gap in terms of new publications, given their low precision in coronary angiographies, whose quality is generally far from optimal. 
In the state of the art review, approximately 50 articles were analysed, based on different focuses and methodologies. Based on these reviews performed by different research groups, we concluded that, among all the algorithms published up until now, Poon's multiscale method [7] provides the highest sensitivity and specificity values for the segmentations performed using it. Furthermore, this algorithm has been used to carry out arterial segmentation in different medical imaging modalities related to angiographies, such as in the case of angiographies of vessels in the retina or in the recording of cerebral vessels [13]. Therefore, it will be used as a reference point to explain our automatic multiscale algorithm proposal for the segmentation of vessels (vascular) in coronary angiography imaging. Its greatest deficiency is relative to the processing times, since it is a semi-automatic method.

The Poon et al. method [7], used as a starting algorithm, is based on multiscale filters to obtain the cost associated with each pixel of the image. The user must indicate the starting and end point of a vascular segment, and the algorithm automatically selects the required intermediate points between both points to outline the vessel. To do so, it uses an exhaustive graphical search based on Dijkstra's algorithm [8]. This method also offers the added control of manual segmentation, allowing the user to only segment the area of the angiography that he/she desires. This is particularly useful when the doctor wishes to focus his/her analysis on a specific area of the image.

The first step of the method is to create the cost matrix of the angiography. Optimization in Poon's et al. is achieved by minimizing the cumulative cost function at each $(x, y, z)$ node. Thus, in this approach the cost associated from a node of a vascular segment $q=(x, y, z)$ to a neighboring node $p=\left(x^{\prime}, y^{\prime}, z^{\prime}\right)$ is calculated as:

$$
\operatorname{Cost}(q, p)=w_{1} C_{v}(p)+w_{2} C_{E v}(q, p)+w_{3} C_{I e}(p)+w_{4} C_{R}(q, p)+w_{5} C_{S}(q, p)
$$

This will be used retrospectively to choose the minimum cost path between the two points that the user selects as the start and end points of a vascular segment. The justification for selecting the minimum cost path is simple: the filters are designed to offer the minimum response inside the vessels and a greater response on what is considered the background. The filters that are used in this phase are the multiscale vessel enhancement filters described by Frangi et al. [14], Koller et al. [15] and several structural filters.

In the case of the former, developed by Frangi et al., the noise and background of the image are suppressed while the vessels are enhanced, in accordance with the following function:

$$
C_{v}(q)=v\left(\lambda_{1}, \lambda_{2}\right)=\left\{\begin{array}{cl}
1 & \text { if } \lambda_{2}>0 \\
1-\exp \left(-\frac{R_{\beta}^{2}}{2 \beta^{2}}\right)\left(1-\exp \left(-\frac{T^{2}}{2 c^{2}}\right)\right) & \text { if } \lambda_{2} \leq 0
\end{array}\right.
$$

with $\lambda_{1}$ and $\lambda_{2}$ being the eigenvalues of the Hessian matrix, $\lambda_{1}<\lambda_{2}, R_{b}=\lambda_{1} / \lambda_{2}, T=$ $\sqrt{\lambda_{1}^{2}+\lambda_{2}^{2}}$ and $c$ affect the sensitivity of the filter and have values of 0.5 and 0.3 , respectively.

The second filter is that of the direction, work of Koller et al. [15]. This algorithm is based on a non-linear combination of linear filters. It searches for elongated, symmetrical and linear structures, such as the minimisation of response at the edges of these structures. This filter creates highly intense coloured lines in the middle of these 
structures, along their entire length. Its value is calculated according to the following mathematical expression:

$$
C_{E v}(q, p)=\frac{2}{\pi} \arccos \frac{E_{v}(p) \cdot E_{v}(q)}{\left|E_{v}(p)\right|\left|E_{v}(q)\right|}
$$

with $E_{v}(i)$ being the eigenvector with the $k$ th smallest magnitude of the Hessian matrix in point $i$. 
Lastly, the structural filtering must be mentioned, which tends to favour the pixels that are found in the middle of the vessels. To do so, it uses different operators, such as the Canny edge detector [16], the gradient of the image [17] and the Laplacian of Gaussian Matrix [18]. The average response of these three operators is called $R(x, y)$. Representing $r$ as the scale, for each $q=(x, y, r)$ analysed, its $E_{v}(x, y, r)$ and $R(x, y)$ are combined to thus define a marker of how centred the point in question is inside of a vessel. This filter then calculates the $R(x, y)$ value at $N$ points, including these points and all the adjacent and normal $\left(P_{R}=\left(x_{i}, y_{i}\right) ; i=1,2, \ldots, N\right)$ to the node $q$. The final cost of the structural filter is defined this way, in order to minimise a response to noise, such as:

$$
C_{I e}(q)=1-\left(\frac{1}{N}\right) \sum_{i=1}^{N} R\left(P_{R}\right)
$$

In addition to these three filters, the algorithm uses two restrictions to detect the best path between the two points. The first is the spatial restriction, which sums a small constant value to the cost of the path for each additional pixel added to it. This way, the distance between the points on the path tends to be smaller, thus avoiding the see-saw effect on the edges of the vessels. For example, the cost that this restriction applies to a path that starts at point $q=(x, y, r)$ and that ends at point $p=\left(x^{\prime}, y^{\prime}, r^{\prime}\right)$ is:

$$
C_{S}(q, p)=\sqrt{\left(x-x^{\prime}\right)^{2}-\left(y-y^{\prime}\right)^{2}}
$$

The algorithm also applies a radius restriction, which penalises the paths on which sudden radius changes appear. This occurs for two reasons. The first of these is that the vessel enhancement filter is sensitive to noise and that it is not reliable to base the radius calculation of the arteries on only the output of this filter. The second is that the radius of the vessels does not tend to suddenly change, unless there is a strong stenosis. Therefore, adding this restriction ensures that the chosen path has progressive radius variations. The equality that defines this restriction is the following:

$$
C_{R}(q, p)=\frac{\left|r-r^{\prime}\right|}{r_{\max }-r_{\min }}
$$

where $r_{\max }$ and $r_{\min }$ are the maximum and minimum possible values of the radius $r$.

Finally, to compute the optimal path in the $(x, y, r)$ space, the cost function is used as the input of a Dijkstra's algorithm [8], a deterministic and exhaustive graph search algorithm that always finds the global optimal path [7].

The aim of this work is to achieve a new automatic method that offers results comparable to the best existing semi-automatic method.

\section{Automatic multiscale algorithm proposal}

The algorithm proposed in this paper uses the Poon et al. method as a starting point, always taking into consideration that this method was initially designed for the segmentation of fundus photography in colour. For coronary angiographies, the images obtained are monochromatic, which minimises the necessary number of colour channels. Based on an initial angiography, the segmentation process starts with a transformation into an HSL colour model, subsequently discarding the hue and saturation channels. The luminance channel, on the other hand, creates the image used in the filtering process. 
Recall that Poon et al. filtered the image on different scales by means of the methods of Frangi et al. and Koller et al., and subsequently applied the Canny and LoG filters, as well as radius and variation restrictions, to segment the vascular vessels. After considering the results of the filters, a cost matrix was obtained that was subsequently used by applying Dijkstra's method, by which the shortest path was established, determining the edges of the identified vessel. From this point on, human intervention was necessary to manually determine two points on the image and for the algorithm to perform the search and definition of the coronary tree. As was previously mentioned, one of the problems that the Poon method presents is that it uses a manual algorithm. As is described in [7], "our proposed technique was $98.2 \%$ reproducible". On the other hand, the algorithm that we will describe below is independent of an operator and $100 \%$ reproducible and verifiable when dealing with a completely automatised segmentation method.

In the proposed method, the Frangi et al. [14], Koller et al. [15] and Evidence filters are applied on different scales, developing our multiscale input in a different way than that initially proposed by Poon et al. [7]. Moreover, an auxiliary image is determined for each scale, based on the maximum values of all the filters. These images are then weighted to obtain an initial segmentation and are ultimately binarised. A prunning iterative method is then applied in order to eliminate incoherent elements in an automated manner that worsens the subsequent result of the segmentation. The differences between both algorithms are shown in Fig. 1 and the different parts between both algorithms are highlighted (manual and automatic). As can be seen, our proposal, in addition to being completely automatic and not requiring the intervention of a clinical expert, analyses the images by following a multiscale approximation based only on the luminance channel, successively applying the Frangi, Koller and evidence algorithms for each of the different scales (alphas). 


\section{HAMARNEH et al. ALGORITHM}

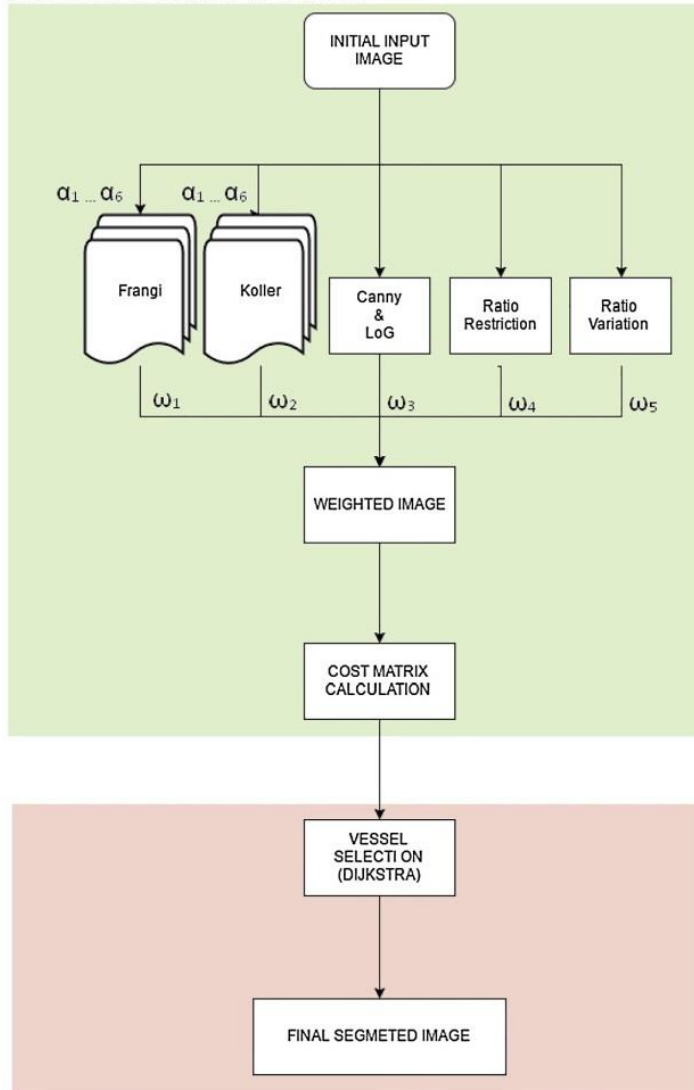

\section{PROPOSED ALGORITHM}

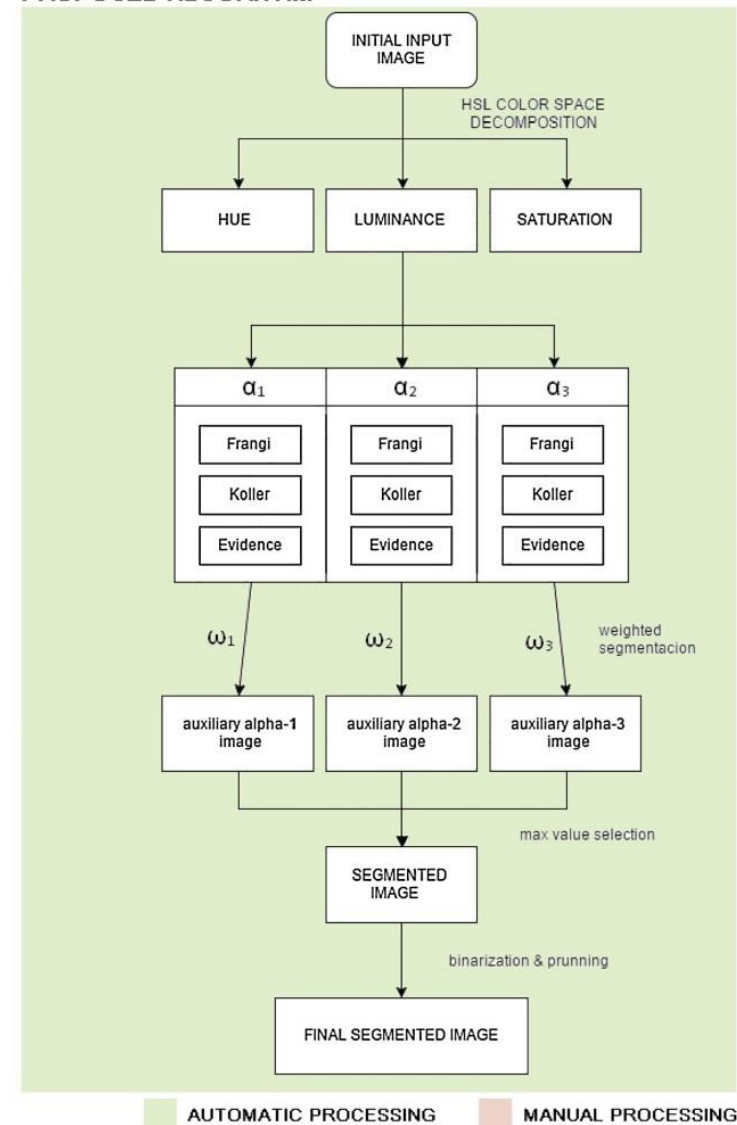

Fig. 1. Workflows related to the Poon et al. algorithms (left) and the proposed method (right). The automatised processes are indicated in green and the manual processes are in red. (For interpretation of the references to color in this figure legend, the reader is referred to the web version of this article.) 
The optimal scales obtained for the coronary angiographies were $[3.5,4.5,5.5]$. In the case of the Frangi et al. filter, the optimal $\beta_{1}$ and $\beta_{2}$ filters obtained were 0.5 and 15.0 , respectively. These values were obtained by means of a leave-one-out crossvalidation [19],[20].

In [7], authors state that their implementation by default uses equal weighting $w_{1-5}=1$ and that varying each weight by $50 \%$ did not change the accuracy by more than $5.2 \%$ using their test images. In our case, a search grid was applied using 30 real angiographies as ground truth, together with the leave-one-out cross-validation to determine and guarantee the adequacy of the values for . Based on the obtained results, the final values used for the optimised version were $\left(w_{1}=0.25 ; w_{2}=0.55 ; w_{3}=\right.$ $0.55)$.

Once the initial segmented image was obtained, a binarisation was performed from the values of the pixels in the image, based on a given cut-off value (threshold). The optimal cut-off value depends on several factors: image brightness, average values of the pixels and pixel variation. The problem with these resulting binarised images lies in the detected artefacts (false positives). In most cases they are bony structures or similar, which can also be detected as erroneous branches from a vessel. In order to minimise this noise, post-processing is applied based on prunning, for which the false positives are minimised due to artefacts or shadows from this type of image.

The operability of the prunning algorithm follows an iterative model: based on the binarised image obtained in the previous step, a convolution model is applied to determine the new active pixels (true positives) of the resulting image. In this case, the calculation is based on determining the number of active pixels in $3 \times 3$ pixel windows. If at least two pixels connected to the reference pixel (the positioned in the center of both axes) are active, it is understood that this pixel in the resulting image will be active.

This process can be considered as a convolutional process over the binarised image using a modified Laplacian operator (including the diagonals). Once the mask is convoluted over the image, a new binarisation threshold process is applied in terms of:

$$
C_{\mathrm{pru}}(p, q)=\left\{\begin{array}{cc}
255 & \text { if } \sum_{u} \sum_{v} f(u, v) g(x-u, y-v)>T \\
0 & \text { otherwise }
\end{array}\right.
$$

where the threshold is the defined as $T=\frac{10}{16}$ and the convolutional kernel as:

$$
\text { Kernel }=\frac{1}{16}\left[\begin{array}{lll}
1 & 1 & 1 \\
1 & 8 & 1 \\
1 & 1 & 1
\end{array}\right]
$$

The idea is that if a pixel is not "connected" to more than one neighbouring pixel (directly or indirectly), it can be considered to be a "loose" pixel. This process is performed as many times as necessary, until no more pixels are altered between iterations.

As shown in Fig. 1, this can be mathematically defined following Poon's approach based on an optimization problem to minimize the cumulative cost function from the node $p$ to the neighboring node $q$ following the next equation:

$$
\operatorname{Cost}(q, p)=C_{\mathrm{pru}}(p, q) *\left(w_{1} C_{v}(p)+w_{2} C_{\mathrm{Ev}}(q, p)+w_{3} C_{\mathrm{Ie}}(p)\right)
$$

Accordingly, the output of the cost function is similar to Fig. $3 \mathrm{~d}$. 
Fig. 2 shows the partial images referring to the application of different multiscale filters, binarisation and the prunnings identified in Fig. 1. In this example the iterative prunning was repeated 32 times. 


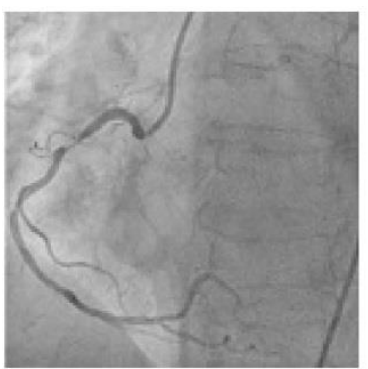

(a)

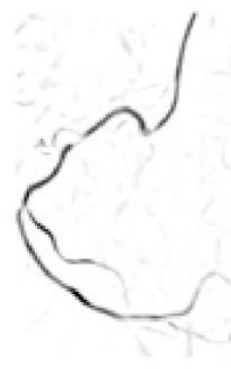

(b)

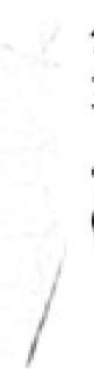

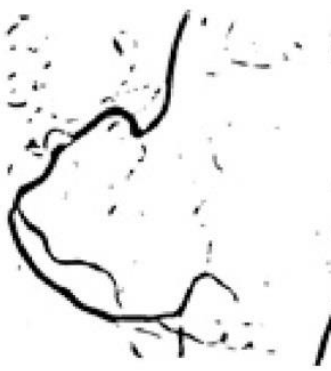

(c)

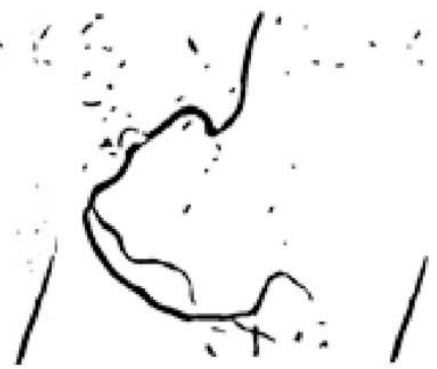

(d)

Fig. 2. Example of the partial images within the workflow shown in Fig. 1. From left to right: original image, prunning post-processed, binarised image and final segmented image obtained by applying the proposed method.

\section{Dataset}

To analyse the efficiency of the presented method, a test set was used that was made up of 30 coronary angiography images, with a $512 \times 512$ pixel resolution, all originating from anonymised angiography studies assigned by the CHUAC Haemodynamics Unit. For each of the images, an ideal segmentation mask was performed to make it possible to verify the segmentation results of the algorithm. This mask was determined by the doctors at the hospital using a manual image editing tool. Several doctors were asked to determine the minimum mask necessary for a correct segmentation (see examples in Fig. 3).

Among the state of the art images, other segmentation images related to coronary angiographies use between 20 and 40 images [21], [22] as test sets, understanding that the set of 30 pairs (original images and ideal masks) can be considered as representational to validate the proposed algorithm. 


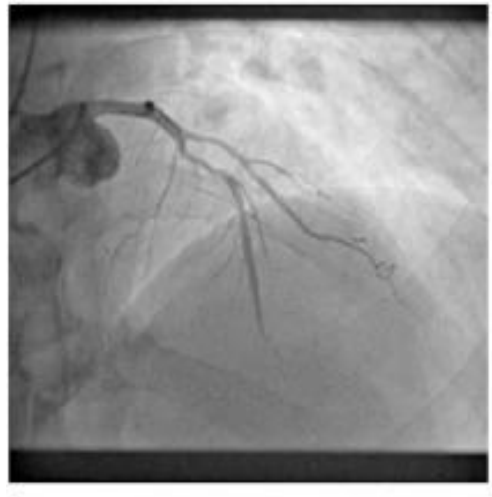

(a)

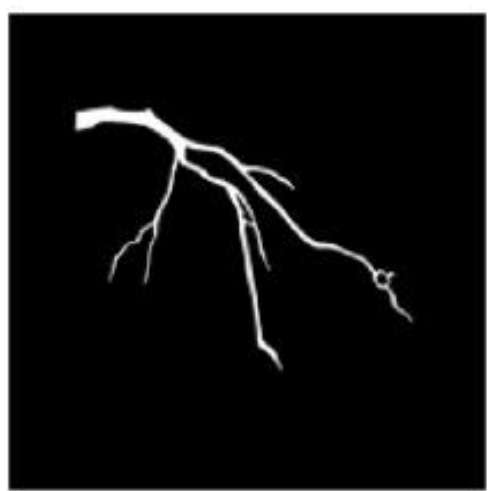

(d)

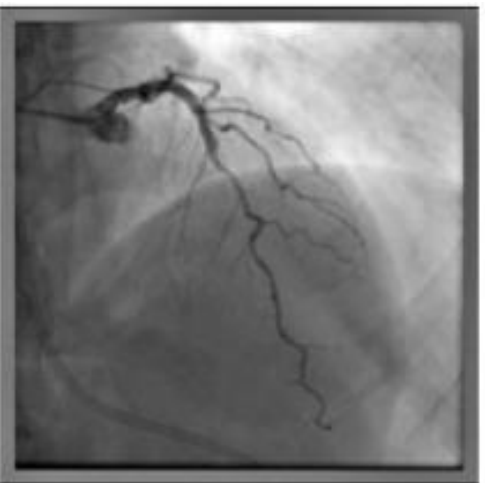

(b)

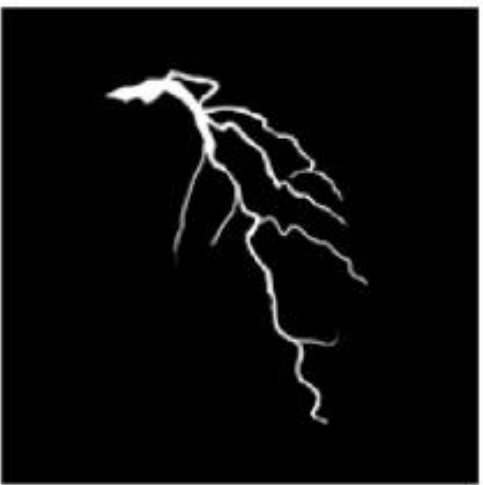

(e)

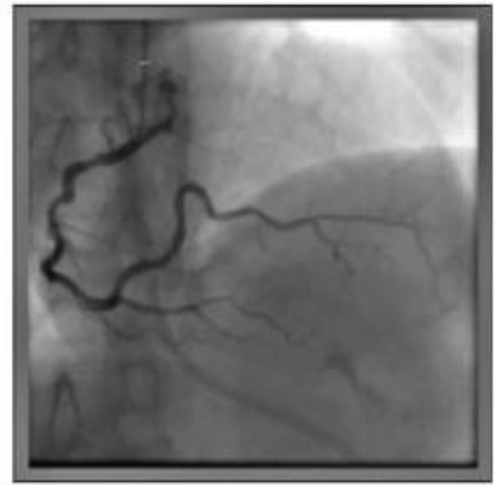

(c)

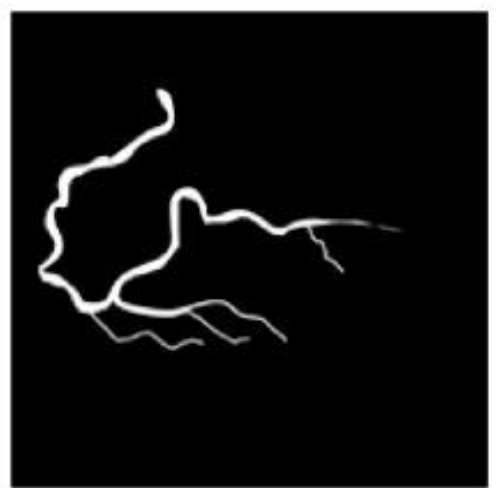

(f)

Fig. 3. Examples of images of the test set. The original angiography images are on the top row. The corresponding ideal masks are on the bottom row.

\section{Results}

In the case of Poon's et al. method, most of the segmentation processing time is spent by the user on the manual selection of the start and end points of each vascular segment that must be segmented, as well as its associated scales. An example of the test cases is shown in Fig. 4. In this image, from left to right, you can see the original image, the ideal mask and the segmentation obtained by the presented algorithm. The three images in each of the 30 cases used in this article are available at https://figshare.com/s/4d24cf3d14bc901a94bf. 


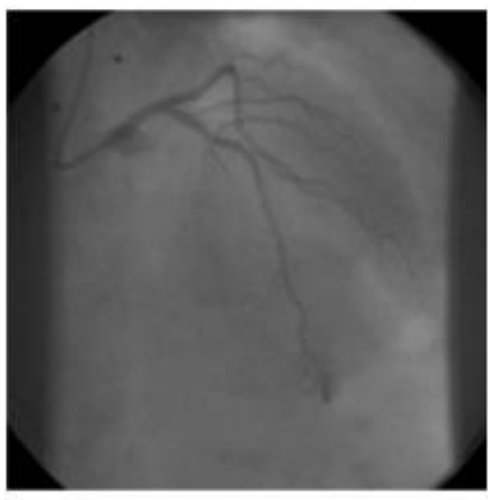

(a)

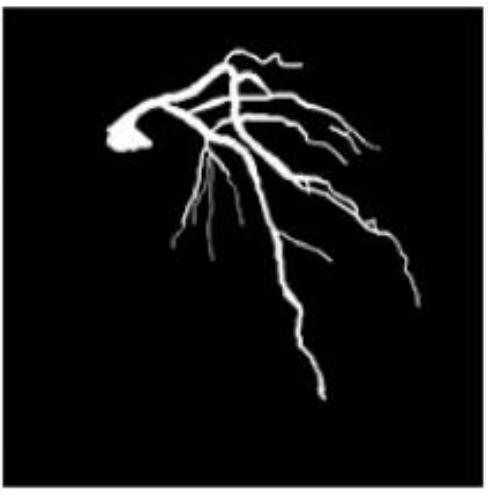

(b)

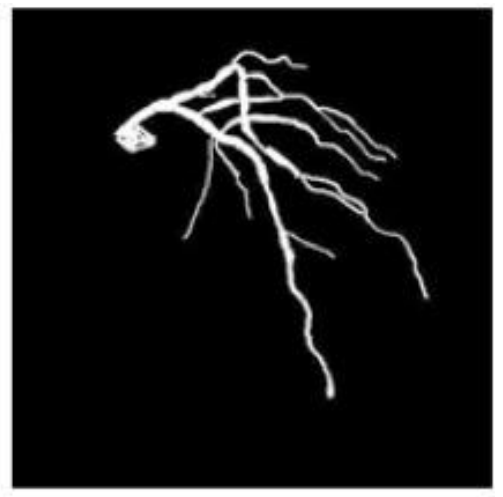

(c)

Fig. 4. Angiography image example, ideal mask and obtained segmentation.

A quantifiable analysis was performed in terms in sensitivity, specificity and the Jaccard index for the segmentations obtained from the designed algorithm with respect to the proposed method. This association of measurements was made in order to cover all the segmented areas in the image. On one side, the sensitivity identifies how many pixels in the object are correctly segmented in the image. On the other side, with the specificity, the number of pixels are calculated that do not belong to the object and are correctly excluded. Lastly, by using the Jaccard index, we collaboratively evaluated the results of the segmentation. In Fig. 5 these values are shown in a boxplot for both methods in each of the 30 test cases and with the three calculated performance measures.

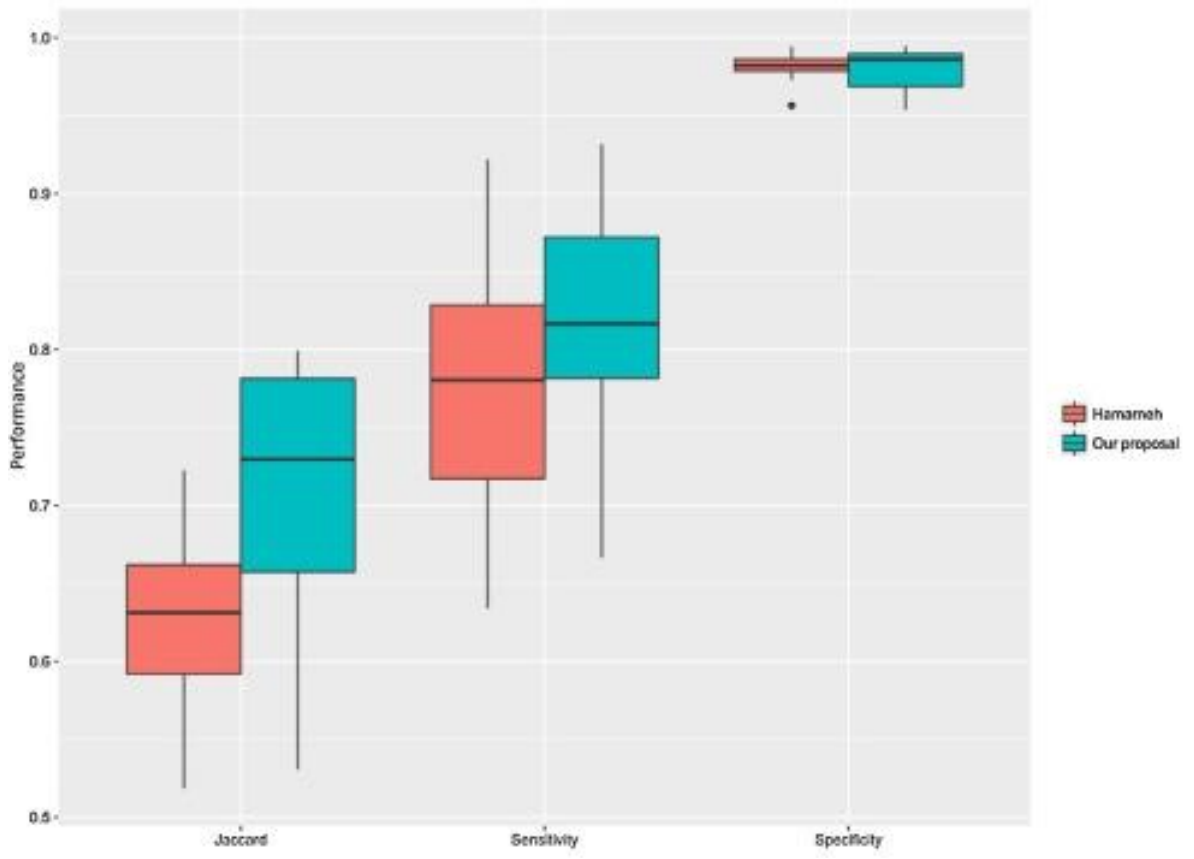


Fig. 5. Boxplot of the Jaccard index, sensitivity and specificity results of the state of the art algorithm proposed by Poon and our proposal for the segmentation of coronary angiographies. 
As can be discerned in Fig. 5, the algorithm shows, overall, high sensitivity and specificity figures and an overall performance very similar to the state of the art algorithm, improved in terms of sensitivity and the Jaccard index [23]. The detection of the image background, i.e., the area that is not a blood vessel, is very high, around $98 \%$, as the specificity indicates, where both algorithms appear to behave very similarly. However, the presented algorithm has less problems when correctly detecting the pixels that represent the vessel, given that the average sensitivity is $82 \%$, compared to $78 \%$ of the original. Our next step was to evaluate the performance of our algorithm by using a widely used performance measurement in imaging segmentation, accepted in the scientific community as the Jaccard index [23]. This measurement was designed to evaluate the performance of cerebral imaging segmentation algorithms due to its simplicity. What is does is measure the intersection ratio of two sets and divides it by its union area.

To compare the two algorithms using the different proposed performance measurements: sensitivity, specificity and the Jaccard index, we followed the method proposed by [24] in the final experimental analysis phase with Machine Learning techniques. To do so, what we first needed to know was, in light of the results, if the statistical test was the most appropriate test to be applied. Although it is intuitively known that the strength of a statistical test may be greater than others, this strength is lost if this test is applied to data that does not meet certain conditions. In light of this, it is necessary to verify if the results obtained by both algorithms, in terms of each of the performance measurements, meet the independence, normality and heteroscedasticity criteria. If any of these three criteria are not met, we must use a nonparametric test, which in this case would be for two Machine Learning models, from which we obtained the performance measurements on a set of 30 images. All the statistical tests that we performed were done with a significance level of $\alpha<0.05$. For clarity reasons, the statistical analysis workflow is schematically summarized in Fig. 6. 


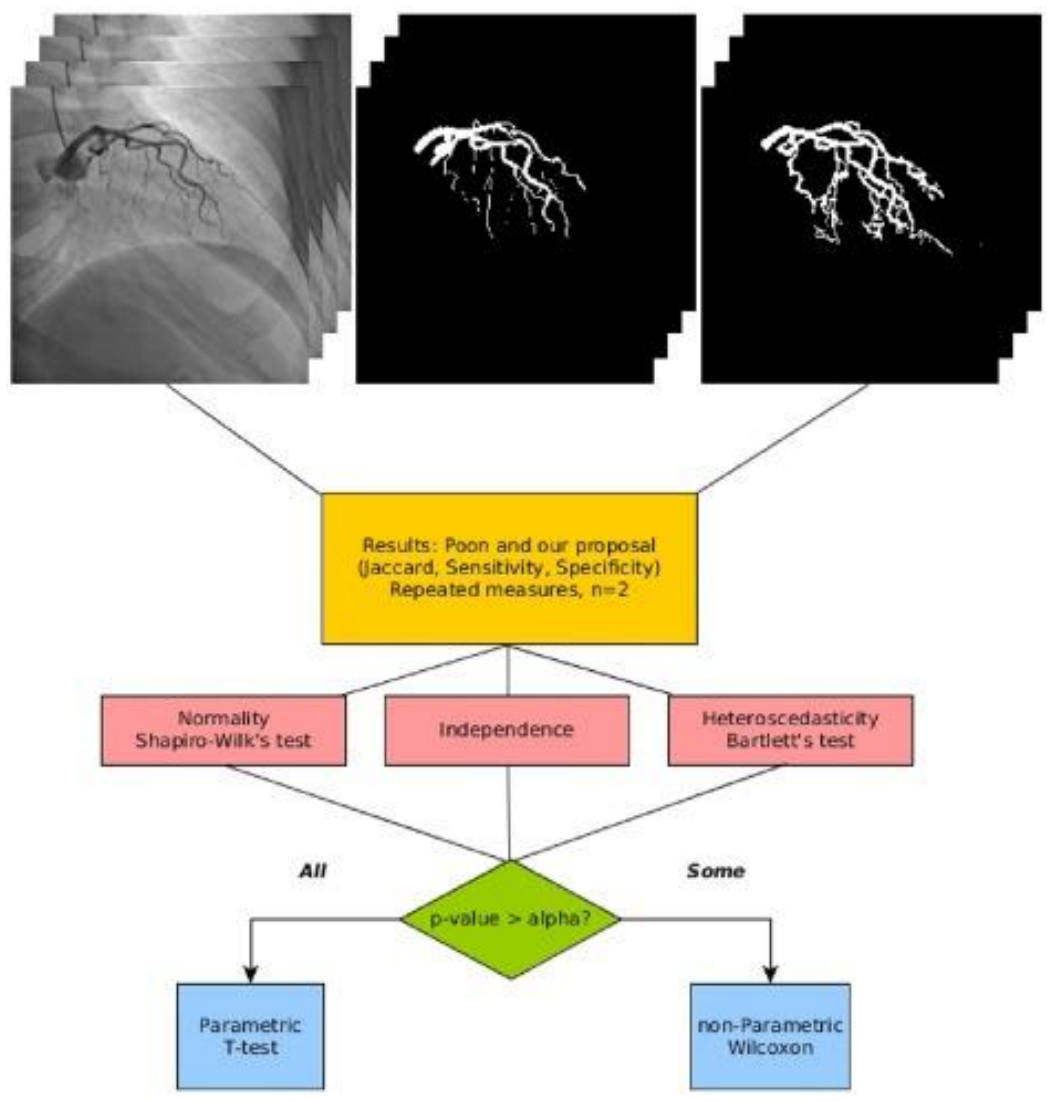

Fig. 6. Workflow for the statistical analysis used in this work. 
We used a Shapiro-Wilk test [25] with the null hypothesis that our results, with respect to the Specificity, would follow a normal distribution. We obtained a $p$-value of $8.073 \mathrm{e}-05$ and $W=0.8941$. Once this result was obtained, it was confirmed that we needed to use a nonparametric test to compare the two models. Therefore, we applied the Wilcoxon test for pairs (pairwise Wilcoxon) [26] with the null hypothesis that both methods would have the same performance and obtain a $p$-value $=0.6263$ i.e., we can affirm with great confidence that there no significant difference between using either of the two algorithms. We used a Shapiro-Wilk test [25] with the null hypothesis that our results, with respect to the Specificity, would follow a normal distribution. We obtained a $p$-vale of 0.3532 and $W=0.97809$, so we could not reject the null hypothesis. We then performed a Bartlett test with the null hypothesis that our results would be homoscedastic. We obtained a Bartlett's $K$ of 1.3381 and a $p$-value of 0.2474 , so we could not reject the null hypothesis again. Once these results were obtained, it was confirmed that we needed to use a parametric test to compare the two models. Therefore, we applied a Welch two samples $T$-test, with the null hypothesis that both methods would have the same performance and obtain a $p$-value $=0.02248$ i.e., we can affirm that the algorithm proposed in this study is significantly better in terms of the Sensitivity performance measurement than that originally proposed in the state of the art. Fig. 7 graphically shows the errors referring to the false positives (specificity) marked in red and the false negatives (sensitivity) marked in green.

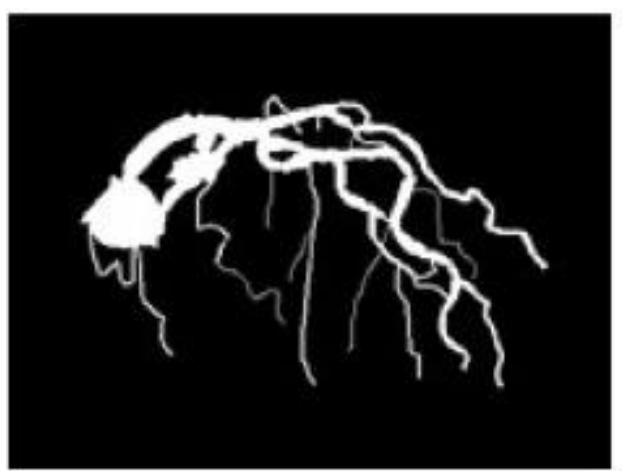

(a)

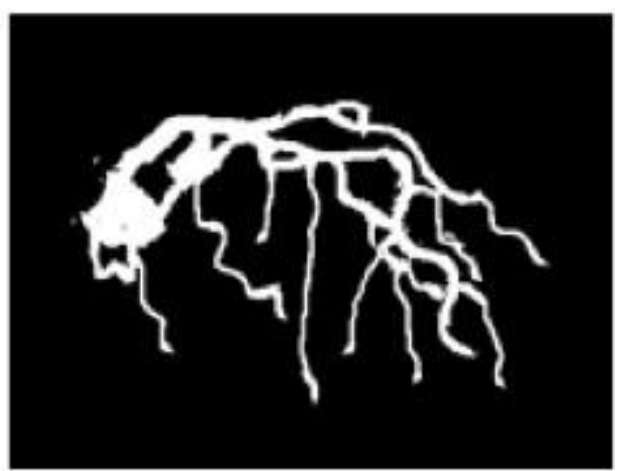

(b)

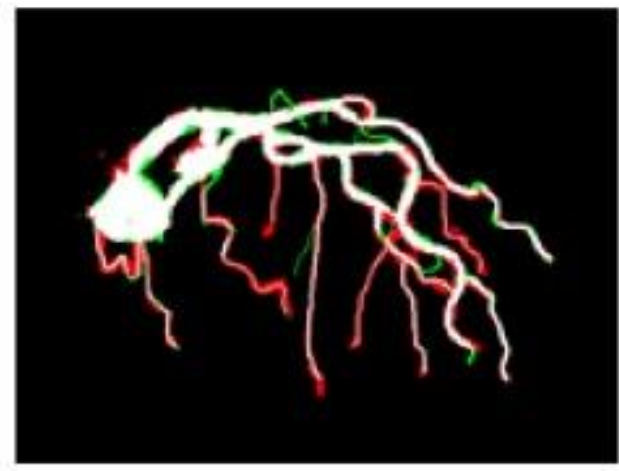

(c)

Fig. 7. Matching comparison between the expected (a) and obtained (b) segmentations. Image (c) identifies true positives in white, true negatives in black, false positives (falsely classified pixels as background) in red 
and false negatives (falsely classified pixels as vessels) in green. (For interpretation of the references to color in this figure legend, the reader is referred to the web version of this article.) 
We used a Shapiro-Wilk test [25] with the null hypothesis that our results, with respect to the Specificity, would follow a normal distribution. We obtained a $p$-vale of 0.01713 and $W=0.95088$. Once this result was obtained, it was confirmed that we needed to use a nonparametric test to compare the two models. Therefore, we applied the Wilcoxon test for pairs (pairwise Wilcoxon) [26] with the null hypothesis that both methods would have the same performance and reject a verify significant $p$ value $=9.313 \mathrm{e}-09$ i.e., we can affirm with great certainty that the algorithm proposed in this study is significantly better in terms of the Jaccard index performance measurement than that originally proposed in the state of the art.

\subsection{Time optimization}

The most expensive operation at the computational level of the presented algorithm is that related to applying the Frangi et al. filter, since it is necessary to obtain the eigenvalues of the matrix that represent the displayed images. The calculation time is to the order of $4.88 \mathrm{~s}$ per radius on average using INTEL i7 $4790 \mathrm{k} 4.00 \mathrm{GHz}$ ( 4 cores, 8 threads) on $512 \times 512$ images. For clarification, the average time spent to perform the manual segmentation of the 30 images used as a test set was $37.29 \mathrm{~s}$. Start and end points in this case where determined by doctor assigned by the CHUAC Haemodynamics Unit. By contrast, the proposed method, by eliminating human interaction, together with a parallel implementation, offers an average segmentation time of $5.69 \mathrm{~s}$ with a standard deviation of $0.64 \mathrm{~s}$.

The presented method is 5 times faster than that presented by Poon et al. This, in absolute terms, has passed the $37.29 \mathrm{~s}$ that the Poon et al. [7] method entails for $512 \times 512$ images, to $5.69 \mathrm{~s}$ that the proposed algorithm uses. This is particularly important considering that, with the current workload level that doctors support during their daily assistance service, the more automatic the system is and the least amount of time required for the segmentation of the coronary tree, the greater the degree of implementation of the algorithm in the national health care system.

In the last column of Table 1 the processing times of each one of the angiographies studied are shown. It is important to bear in mind that in all cases these times are less than those by the most expensive scale of Poon's et al. approach. That is to say, the simplification and automation of the method, together with its multi-thread implementation, offers a significant improvement in processing times. 
Table 1. Differences between the proposed and Poon's et al. algorithm in terms of execution time. From left to right, the total times of Poon's approach are shown, time used by the most expensive computationally scale, the total time used in the automatic part of the method and by the manual part. Finally, the time used by the proposed automatic algorithm is shown.

\begin{tabular}{|c|c|c|c|c|c|}
\hline & \multicolumn{4}{|c|}{ Poon's implementation } & \multirow[t]{2}{*}{ Proposed algorithm } \\
\hline & Total time & Slowest scale & All scales & Manual & \\
\hline Angio \#1 & 30,052 & 9685 & 14,528 & 15,524 & 5075 \\
\hline Angio \#2 & 34,567 & 9763 & 14,645 & 19,922 & 5401 \\
\hline Angio \#3 & 33,973 & 9767 & 14,650 & 19,323 & 5120 \\
\hline Angio \#4 & 29,045 & 9671 & 14,506 & 14,539 & 5846 \\
\hline Angio \#5 & 33,743 & 9729 & 14,593 & 19,150 & 5747 \\
\hline Angio \#6 & 29,979 & 9704 & 14,556 & 15,423 & 5483 \\
\hline Angio \#7 & 51,745 & 9814 & 14,721 & 37,024 & 6634 \\
\hline Angio \#8 & 65,104 & 9863 & 14,794 & 50,310 & 6027 \\
\hline Angio \#9 & 33,617 & 9796 & 14,695 & 18,922 & 6576 \\
\hline Angio \#10 & 37,238 & 9808 & 14,713 & 22,525 & 5913 \\
\hline Angio \#11 & 38,271 & 9803 & 14,705 & 23,566 & 5985 \\
\hline Angio \#12 & 34,484 & 9783 & 14,674 & 19,810 & 3823 \\
\hline Angio \#13 & 43,243 & 9836 & 14,754 & 28,490 & 5881 \\
\hline Angio \#14 & 44,489 & 9848 & 14,772 & 29,717 & 5854 \\
\hline Angio \#15 & 49,745 & 9853 & 14,779 & 34,966 & 6142 \\
\hline Angio \#16 & 74,264 & 9880 & 14,821 & 59,443 & 5962 \\
\hline Angio \#17 & 35,702 & 9781 & 14,671 & 21,030 & 5982 \\
\hline Angio \#18 & 35,455 & 9798 & 14,697 & 20,758 & 5279 \\
\hline Angio \#19 & 34,606 & 9761 & 14,642 & 19,965 & 5988 \\
\hline Angio \#20 & 33,391 & 9717 & 14,575 & 18,815 & 6201 \\
\hline Angio \#21 & 35,393 & 9787 & 14,681 & 20,712 & 6051 \\
\hline Angio \#22 & 32,907 & 9751 & 14,626 & 18,281 & 6161 \\
\hline Angio \#23 & 30,831 & 9731 & 14,597 & 16,234 & 5539 \\
\hline Angio \#24 & 30,256 & 9716 & 14,573 & 15,682 & 4239 \\
\hline Angio \#25 & 36,960 & 9745 & 14,617 & 22,343 & 6397 \\
\hline Angio \#26 & 31,664 & 9726 & 14,589 & 17,076 & 5579 \\
\hline Angio \#27 & 28,718 & 9733 & 14,600 & 14,118 & 4610 \\
\hline Angio \#28 & 33,132 & 9759 & 14,638 & 18,494 & 5645 \\
\hline Angio \#29 & 30,142 & 9682 & 14,523 & 15,619 & 5554 \\
\hline Angio \#30 & 25,985 & 9511 & 14,266 & 11,718 & 6004 \\
\hline
\end{tabular}

Therefore, it has been statistically proven that our algorithm, in less time and eliminating the human factor, is able to obtain results statistically equivalent to those of the state of the art in terms of specificity. The statistical test indicates that there is no significant difference between them and that it clearly surpasses it in terms of sensitivity and the Jaccard index, since the statistical test indicates, with a very significant value, that there are differences between the compared techniques. 


\section{Discussion}

To ensure that our results are relevant, we have tested, through different parametric and nonparametric statistical contrast tests of the null hypothesis, that our algorithm is statistically and significantly better than that previous published in the state of the art. The performance measurements in which the study is statistically better are: sensitivity and the Jaccard index. We have also proven that there is no significant difference in using either of the two algorithms if the comparison criterion is its specificity. In this sense, we wish to emphasise that the use of Dijkstra's algorithm in the final part of Poon's et al. algorithm implies that the user manually determines which parts of the coronary tree are accepted. On the other hand, in the proposal of this article, since there is no human interaction in any process, it is the algorithm that automatically segments any initial angiography image, with a consequent saving of time and suppression of inter- and intra-operator variability.

It is also worth mentioning that the time required to perform each segmentation using other semi-automatic methods depends entirely on the ability of the user and the quantity of blood vessels wished to be segmented. This process, in addition to being difficult to reproduce and dependent on the experience of the clinician performing the task, is critical if dealing with a system applicable to daily practice and its implementation in a haematology service. Thanks to the algorithm proposed in this study, the segmentation times and the results obtained are $100 \%$ reproducible in a shorter time interval and prevent biases due to human intervention.

If we focus on the results obtained by the proposed algorithm, it is important to stress that the precision obtained in wide vessels is excellent, considering the data provided by the statistical markers. The data obtained in narrow vessels is also very good, although it can be observed that it is slightly less than that of the wide vessels, according to the metric values obtained in these areas. Our results also highlight that the sensitivity to noise of the proposed system is minimal due to the use of a set of several independent techniques to create the final segmented image.

Moreover, the results obtained in this work are similar to other recent studies in the field using different machine learning approaches. The best performance is achieved in the Nasr-Esfahani et al. work [27] using convolutional nets with an average specificity of $98.59 \%$ and sensitivity of $86.76 \%$ over a set of 44 angiographies of size $512 \times 512$ pixels. In addition, the overall processing time per image is $106 \mathrm{~s}$. In Khowaja et al. [28] the authors states that their method offers the best performance with the minimum processing time with a specificity of $98.03 \%$ and a sensitivity of $70.28 \%$ over 20 DICOM images of identical nature in $2.2 \mathrm{~s}$ average.

Lastly, in the presented method, as was seen in the exhaustive statistical analysis performed, there is a clear existence of errors produced by false positives, which, as we have been able to see, tend to be related to the "width" of the detected arteries. This problem is related to the impossibility of using variable width radii. It is because of this that, in the near future, we intend to study if it is possible develop a new multiscale module where the radii have variable widths in order to minimise the influence of the widths of the vessels. Furthermore, we found another problem related to the final convolution with the modified kernel based on the Laplacian operator; in those cases within the limits of the coronary tree the result was a false negative, especially in branches with few pixels of width. 


\section{Conclusions}

This article proposes a new automatic multiscale segmentation algorithm for coronary angiography images for the study of coronary trees. A design of the experiment has been created that makes it possible to validate our development and to pit it against some of the current state of the art algorithm, according to different, already published state of the art reviews and the literature search performed by the authors. What is more, based on this state of the art algorithm, we have researched a different approach that involves optimisation, and that has been able to statistically prove that the study we developed is better, basing this affirmation on different success measurements. We used a representative number of coronary angiography images that was greater than the minimum established by previous publications to ensure the generalisation capacity of the algorithm.

The algorithm presented has been described in detail throughout this article and the most remarkable conclusion is that our study can be used as an automatic segmentation system for coronary angiographies in daily assistance services. This is possible due to its outstanding precision and its drastic reduction in the calculation time required to segment images of this nature and, ultimately, to present the segmentation results to the doctors. These improvements to the algorithm imply that the clinician can perform tests on the patient and, by passing the images through the system, can verify, in that moment, the intervention of existing differences in a coronary tree from a previous test, in such a way that it could change its clinical intra-intervention criteria.

\section{Acknowledgements}

This work was supported in part by the General Directorate of Culture, Education and University Management of Xunta de Galicia (Ref. GRC2014/049), the Ministry of Economy and Competitiveness of Spain (Project TIN2015-70648-P) and the Juan de la Cierva fellowship program by the Spanish Ministry of Economy and Competitiveness (Carlos Fernandez-Lozano, Ref. FJCI-2015-26071).

\section{References}

[1] World Health Organization, http://www.who.int/mediacentre/factsheets/fs317/es/index.html (accessed: 2010-11-20).

[2] American Heart Association, http://www.americanheart.org/presenter.jhtml?identifier=4478 (accessed: 2010-10-25).

[3] European Heart Network, European Cardiovascular Disease Statistics, http://www.ehnheart.org/files/statistics2008web-161229A.pdf (accessed: 2010-11-01).

[4] J. Brien, J.F. O'brien, N.F. EzquerraAutomated segmentation of coronary vessels in angiographic image sequences utilizing temporal, spatial and structural constraints. Proceedings of SPIE Visualization in Biomedical Computing Spatial and Structural Constraints (1994), pp. 25-37

[5] C. Kirbas, F. QuekA review of vessel extraction techniques and algorithms. ACM Comput. Surv., 36 (2) (2004), pp. 81-121, 10.1145/1031120.1031121

[6] J. Novoa, N. Ezquerra, L. Traba, M. Villar, J. Pereira, J. Manuel Vazquez-Rodriguez, N. Vazquez, M. Martinez-Romero, V. MatoExtraction of quantitative anatomical information from coronary angiographies. Curr. Bioinform., 6 (2) (2011), pp. 233-250

[7] K. Poon, G. Hamarneh, R. AbugharbiehLive-Vessel: Extending Livewire for Simultaneous Extraction of Optimal Medial and Boundary Paths in Vascular Images. Springer Berlin Heidelberg, Berlin, Heidelberg (2007), pp. 444-451, 10.1007/978-3-540-75759-7_54

[8] E.W. DijkstraA note on two problems in connexion with graphs. Numer. Math., 1 (1) (1959), pp. 269-271, 10.1007/BF01386390 
[9] L. GradyRandom walks for image segmentation. IEEE Trans. Pattern Anal. Mach. Intell., 28 (11) (2006), pp. 1768-1783, 10.1109/TPAMI.2006.233

[10] Y. YangImage Segmentation and Shape Analysis of Blood Vessels with Applications to Coronary Atherosclerosis (Ph.D. thesis). Georgia Institute of Technology (2007)

[11] R. Nekovei, Y. SunBack-propagation network and its configuration for blood vessel detection in angiograms. IEEE Trans. Neural Netw., 6 (1) (1995), pp. 64-72

[12] M. Karapataki, P. De WildeHopfield network applied to blood vessel detection in angiograms

Med. Biol. Eng. Comput., 35 (4) (1997), pp. 428-430

[13] F. Pernus, et al.3d-2d registration of cerebral angiograms: a method and evaluation on clinical images. IEEE Trans. Med. Imaging, 32 (8) (2013), pp. 1550-1563

[14] A.F. Frangi, W.J. Niessen, K.L. Vincken, M.A. ViergeverMultiscale Vessel Enhancement Filtering. Springer Berlin Heidelberg, Berlin, Heidelberg (1998), pp. 130-137, 10.1007/BFb0056195

[15] T.M. Koller, G. Gerig, G. Szekely, D. DettwilerMultiscale detection of curvilinear structures in 2-d and 3-d image data. Proceedings of IEEE International Conference on Computer Vision (1995), pp. 864-869, 10.1109/ICCV.1995.466846

[16] J. CannyA computational approach to edge detection. IEEE Trans. Pattern Anal. Mach. Intell. (1986), pp. 679-698, 10.1109/TPAMI.1986.4767851

[17] D. WangA multiscale gradient algorithm for image segmentation using watershelds. Pattern Recognit., 30 (12) (1997), pp. 2043-2052

[18] Spatial Filters - Laplacian of Gaussian, http://homepages.inf.ed.ac.uk/rbf/HIPR2/log.htm (accessed: 2010-12-13).

[19] P. Refaeilzadeh, L. Tang, H. LiuCross-validation. Encyclopedia of Database Systems, Springer (2009), pp. 532-538

[20] S. Arlot, A. Celisse, et al. A survey of cross-validation procedures for model selection. Stat. Surv., 4 (2010), pp. 40-79

[21] Y.-C. Tsai, H.-J. Lee, M.Y.-C. ChenAutomatic segmentation of vessels from angiogram sequences using adaptive feature transformation. Comput. Biol. Med., 62 (2015), pp. 239-253

[22] F. Cervantes-Sanchez, I. Cruz-Aceves, A. Hernandez-Aguirre, J.G. Avi na-Cervantes, S. Solorio-Meza, M. Ornelas-Rodriguez, M. Torres-CisnerosSegmentation of coronary angiograms using Gabor filters and Boltzmann univariate marginal distribution algorithm. Comput. Intell. Neurosci. (2016)

[23] P. JaccardThe distribution of the flora in the alpine zone. New Phytol., 11 (2) (1912), pp. $37-$ 50

[24] C. Fernandez-Lozano, M. Gestal, C.R. Munteanu, J. Dorado, A. PazosA methodology for the design of experiments in computational intelligence with multiple regression models. PeerJ, 4 (2016), p. e2721

[25] S.S. Shapiro, M.B. WilkAn analysis of variance test for normality (complete samples). Biometrika, 52 (3-4) (1965), pp. 591-611

[26] F. WilcoxonIndividual comparisons by ranking methods. Biom. Bull., 1 (6) (1945), pp. 8083

[27] E. Nasr-Esfahani, N. Karimi, M. Jafari, S. Soroushmehr, S. Samavi, B. Nallamothu, K. NajarianSegmentation of vessels in angiograms using convolutional neural networks. Biomed. Signal Process. Control, 40 (2018), pp. 240-251, 10.1016/j.bspc.2017.09.012

[28] S. Khowaja, M. Unar, I. Ali Ismaili, P. KhuwajaSupervised method for blood vessel segmentation from coronary angiogram images using 7-d feature vector. Imaging Sci. J., 64 (2016), pp. 196-203 